\title{
The results of delayed open reduction and internal fixation in chronic bony mallet finger injuries
}

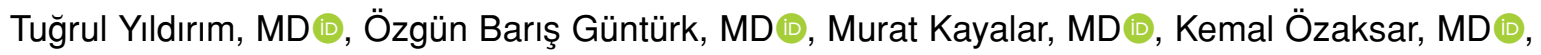 \\ Tahir Sadık Sügün, MD®D, Yalçın Ademoğlu, MD (D) \\ Department of Orthopedics and Traumatology, Hand Microsurgery, Emot Hospital, Izmir, Turkey
}

Mallet finger is a zone 1 extensor injury that occurs in the form of tendon rupture or avulsion fracture at the distal phalanx base and the injury is caused by traction of the terminal extensor tendon. ${ }^{[1]}$ Although most cases can be treated conservatively with a splint, surgery is indicated for the bony mallet finger, when the distal interphalangeal (DIP) joint is subluxated or when the fracture fragment is greater than $30 \%$ of the joint surface. ${ }^{[1,2]}$ The main goals of treatment are to restore the joint congruity, minimize the extensor lag, and maintain painless motion at the DIP joint. The fixation of the fragment can be performed by various methods, including the use of Kirschner wires (K-wires), tension band wiring, screws, pull-out wires, hook plates, or an external fixator. ${ }^{[3-7]}$

One of the factors affecting the management of mallet finger is timing of hospital admission, specifically acute versus delayed presentation.

Received: May 04, 2020

Accepted: June 11, 2020

Published online: November 19, 2021

Correspondence: Tuğrul Yıldırım, MD. EMOT Hastanesi, Ortopedi ve Travmatoloji Bölümü, 35230 Konak, İzmir, Türkiye.

E-mail: mdtugrul@hotmail.com

Doi: $10.52312 / j d r s .2021 .240$

Citation: Yıldırım T, Güntürk ÖB, Kayalar M, Özaksar K, Sügün TS, Ademoğlu $Y$. The results of delayed open reduction and internal fixation in chronic bony mallet finger injuries. It Dis Relat Surg 2021;32(3):625-632.

(C2021 All right reserved by the Turkish Joint Diseases Foundation

This is an open access article under the terms of the Creative Commons Attribution-NonCommercial License, which permits use, distribution and reproduction in any medium, provided the original work is properly cited and is not used for commercial purposes (http://creativecommons.org/licenses/by-nc/4.0/).

\section{ABSTRACT}

Objectives: The aim of this study was to evaluate the functional results of delayed open reduction and Kirschner wire (K-wire) fixation procedures in patients with delayed presentation of bony mallet finger.

Patients and methods: Between February 2009 and November 2019, a total of 19 patients (15 males, 4 females; median age: 24.8 years; range, 14 to 47 years) who were diagnosed with a delayed bony mallet finger and treated with dorsal block pin, direct pinning, or the umbrella handle technique were retrospectively analyzed. The Crawford criteria were used to evaluate the outcomes. The degrees of range of motion (ROM) were measured by a goniometer.

Results: The median time from injury to surgery was 41 (range, 28 to 90 ) days. The median DIP joint extension limitation was 7.63 (range, 0 to 40 ) degrees and the median ROM of the DIP joint was 66.3 (range, 20 to 90) degrees. There was no statistically significant difference in the postoperative ROM, compared to the uninjured side $(\mathrm{p}>0.05)$. The Crawford score was excellent in 11 , good in four, fair in three, and poor in one patient. Bone union was achieved in all patients.

Conclusion: Delayed open reduction and K-wire fixation of chronic bony mallet finger injuries yield successful functional outcomes with low complication rates. Extension lag can be eliminated in most patients by making the joint surface anatomical. The most optimal method should be selected depending on the size of the fracture fragment.

Keywords: Bony mallet finger, distal interphalangeal joint luxation, hook plate, Ishiguro technique, Wehbé-Schneider classification.

Some patients are admitted to the hospital weeks after the injury, without a diagnosis or with inadequate treatment. Although there is a controversy regarding the threshold value for considering a mallet finger injury delayed, the general definition is four to five weeks after injury. ${ }^{[8,9]}$ Surgical treatment is recommended in these cases, if there is flexion deformity greater than 40 degrees or limited dorsiflexion. ${ }^{[1]}$ Untreated cases 
may result in extension lag, swan neck deformity, or DIP joint degeneration. ${ }^{[10]}$ Anatomical reduction is recommended to prevent joint deformity and secondary arthritis in chronic cases. ${ }^{[11,12]}$

Although many studies in the literature present the results of surgical treatment for acute bony mallet finger, studies including cases with delayed presentations are limited. In delayed cases, the scar tissue formed between the fracture fragments prevents closed reduction; thus, fracture healing and functional results are adversely affected. ${ }^{[13]}$ Although direct reduction of bony fragments can be done with open reduction, small bone fragments can be damaged during manipulation, and skin problems, nail dystrophy, osteoarthritis, stiffness, or extensor lag may develop after open reduction. ${ }^{[14]}$

In the present study, we hypothesized that anatomical restoration of the joint surface would yield similar functional results and complication rates to those treated acutely for a mallet finger. We, therefore, aimed to evaluate the results of different K-wire fixation methods applied according to the size of fracture fragments in patients with delayed presentation of bony mallet finger ( $>4$ weeks) and to identify complications.

\section{PATIENTS AND METHODS}

This single-center, retrospective study was conducted at private Izmir Hand Microsurgery Orthopedics Traumatology Hospital between February 2009 and November 2019. A total of 19 patients (15 males, 4 females; median age: 24.8 years; range, 14 to 47 years) who were diagnosed with a delayed bony mallet finger were included in the study. Patients with a bony fragment involving less than $30 \%$ of the joint surface or with open injuries, malunion, or osteoarthritis in the DIP joint were excluded from the study. A written informed consent was obtained from the patients and/or their legal guardians. The study protocol was approved by the Izmir Hand Microsurgery Orthopedics Traumatology Hospital Ethics Committee (Date/No:05/02/2021/002). The study was conducted in accordance with the principles of the Declaration of Helsinki.

Demographic and clinical data, operative data, time to delay, and the reason for delayed presentation were obtained from the hospital database. Patients who were not treated for $\geq 4$ weeks, who were not treated appropriately, or in whom conservative treatment failed were considered to have delayed presentation. Failure of conservative treatment was defined as the continuation of the extension lag and volar subluxation. Only patients with bony fragments involving more than $30 \%$ of the joint surface or patients with distal phalanx subluxation were included in the study.

The criteria defined by Crawford ${ }^{[15]}$ were used to evaluate mallet finger outcomes. The flexion-extension degree of the corresponding finger on the healthy side was considered as standard. The degrees of loss of extension and flexion were measured by a goniometer.

\section{Surgical technique}

All patients underwent surgery performed by highly-experienced orthopedic hand surgeons. ${ }^{[16]}$ After the regional block, a tourniquet was inflated up to 250 to $270 \mathrm{mmHg}$. An H-shaped incision was made over the DIP joint (Figure 1). As it can be difficult to distinguish the terminal tendon from the soft callus of the fracture fragment, a lateral fluoroscopic view may be helpful to determine
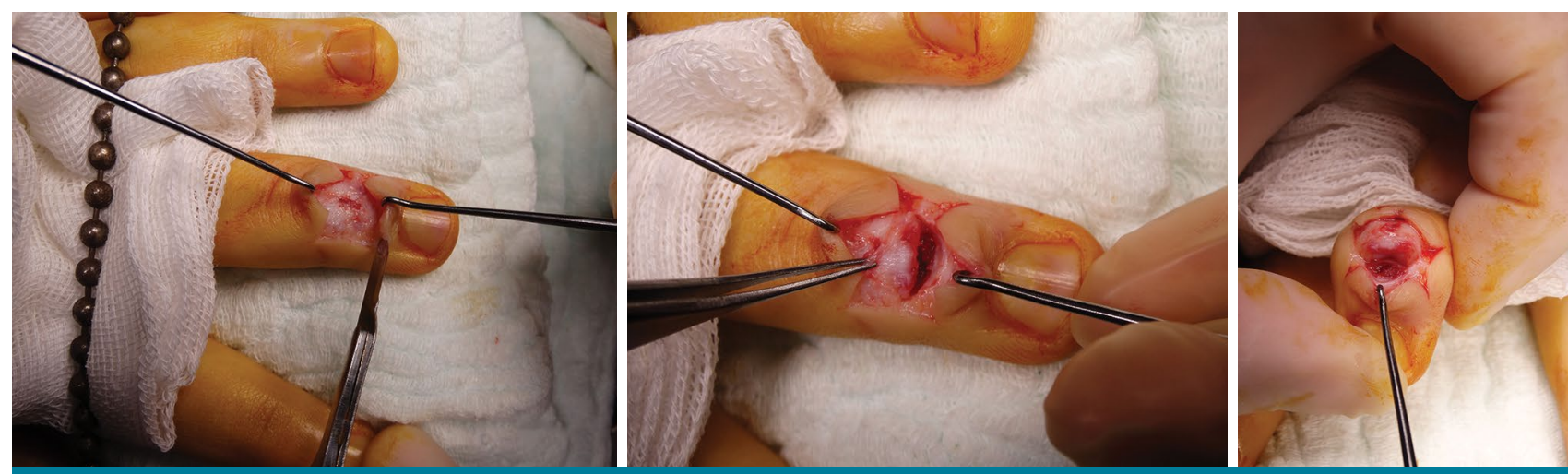

FIGURE 1. An H-shaped incision was made over the distal interphalangeal joint joint. The fibrotic tissue between the fragment and distal phalanx was removed with a curette to expose the fracture surface. 

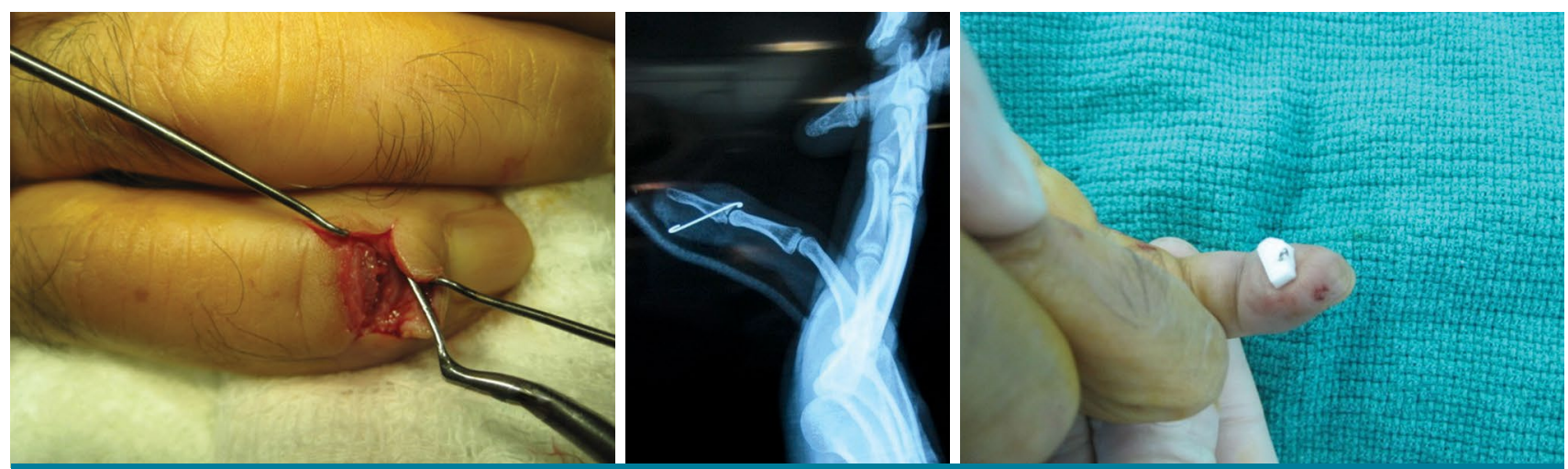

FIGURE 2. In the umbrella handle technique, the dorsal fragment was fixed with a K-wire directed obliquely from a proximal to distal direction. After fixing the fracture, the dorsal end of the K-wire was bent into the shape of an umbrella handle. Umbrella handle was drawn down to reduce the fracture by pulling on the K-wire from the palmar aspect of the finger.

K-wire: Kirschner wire.

the fracture site. The fibrotic tissue between the fragment and distal phalanx was removed with a curette to expose the fracture surface. Manipulating the dorsal fragment with reduction forceps would risk fragmentizing the fracture or disrupting the terminal tendon. The challenge is that the surgeon must correct the rotational deformity and reduce the dorsal fragment, to which the extensor digitorum communis is attached and which contains the articular cartilage. Anatomical reduction cannot be achieved, if rotation of the fragment is present. As there is no chance to directly visualize the congruity of the DIP joint after reduction, the dorsal fragment should be evaluated with a magnified lateral fluoroscopy.

The umbrella handle method was preferred, if the dorsal cortical length of the fragment was small or would be fragmented when fixed directly with a K-wire ${ }^{[17]}$ (Figure 2). In such cases, the dorsal fragment was fixed with a K-wire directed obliquely from a proximal to distal direction. After fixing the fracture, the dorsal end of the K-wire was bent into the shape of an umbrella handle. Then, this umbrella handle was drawn down to reduce the fracture by pulling on the $\mathrm{K}$-wire from the palmar aspect of the finger.

The dorsal block pin (DBP) method by Ishiguro et al. ${ }^{[18]}$ was preferred, when the fragment involved 30 to $40 \%$ of the joint surface (Figure 3). While keeping the DIP joint in maximum flexion, the surgeon inserted a K-wire through the terminal extensor tendon into the middle phalanx, just behind the fragment under lateral-view fluoroscopic imaging. The distal phalanx was, then, pulled distally, and the DIP joint was extended to reduce the fracture. If the reduction provided by the extension block pin was adequate, a second K-wire was inserted into the distal phalanx volar to the fracture line and across the DIP joint to hold it in slight hyperextension.

When the fragment size was close to $50 \%$ of the joint surface, it was difficult to insert the DIP
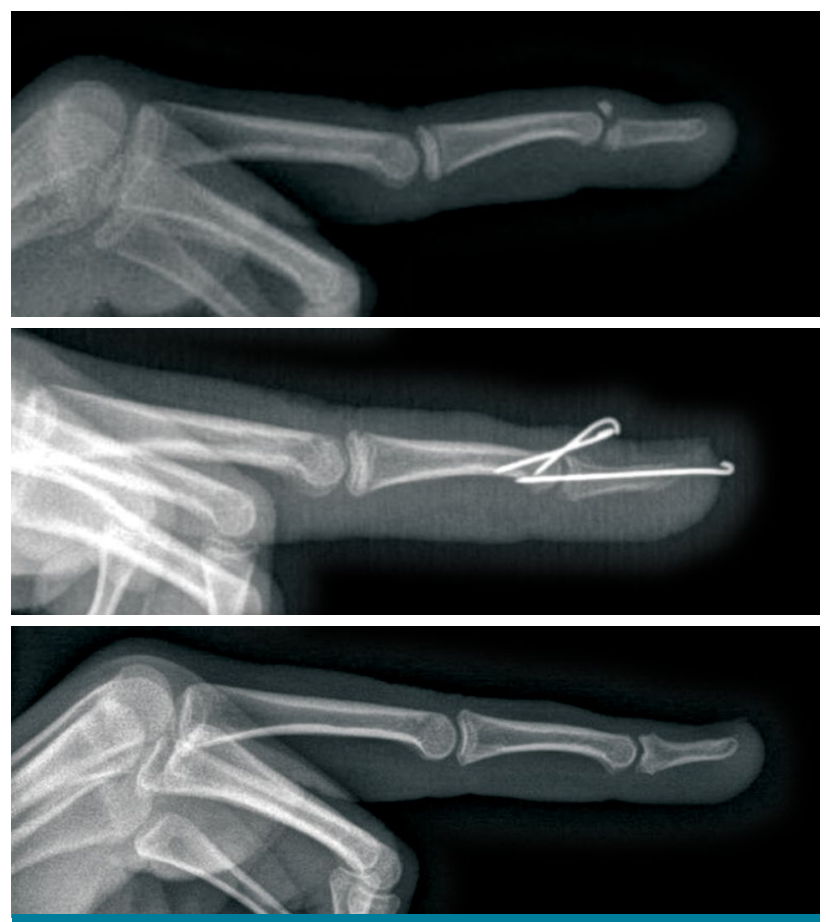

FIGURE 3. The dorsal block pin technique was preferred, when the fragment involved 30 to $40 \%$ of the joint surface. One or two K-wires were inserted through the terminal extensor tendon into the middle phalanx, just behind the fragment under lateral-view fluoroscopic imaging. If the reduction provided by the extension block pin was adequate, a second $\mathrm{K}$-wire was inserted across the distal interphalangeal joint. K-wire: Kirschner wire. 
transfixation wire after DBP, as the wire would coincide with the fracture line. In such cases, the joint could remain subluxated and the fragment could not be reduced. Therefore, in this case, direct fragment fixation, which was described by Badia and Riano, ${ }^{[19]}$ was preferred (Figure 4). For this procedure, the DIP transfixation wire was inserted volarly as much as possible, checking that it was not passing through the fracture line (Figure 5). After DIP transfixation, the fragment was reduced with forceps. However, it would be difficult to observe any incongruency in the joint. Therefore, reduction and any step-off should be checked with a magnified lateral fluoroscopic view. Finally, in the reduced state, the fragment was fixed using a second K-wire inserted from the dorsal to the volar side. Multiple K-wire attempts were avoided to prevent fragmentation and damage to the articular surface. Irrespective of the method used, the dorsal fragment should be compressed and any articular step-off should be avoided after fixation.

\section{Postoperative follow-up}

In all patients, postoperative proximal interphalangeal joint and metacarpophalangeal joint movements were started immediately. No splint or brace was needed after surgery. At the end of six weeks, the K-wires were removed and the patients were referred to the hand therapists. At the final follow-up visit, the presence of union and osteoarthritis were evaluated by radiography.
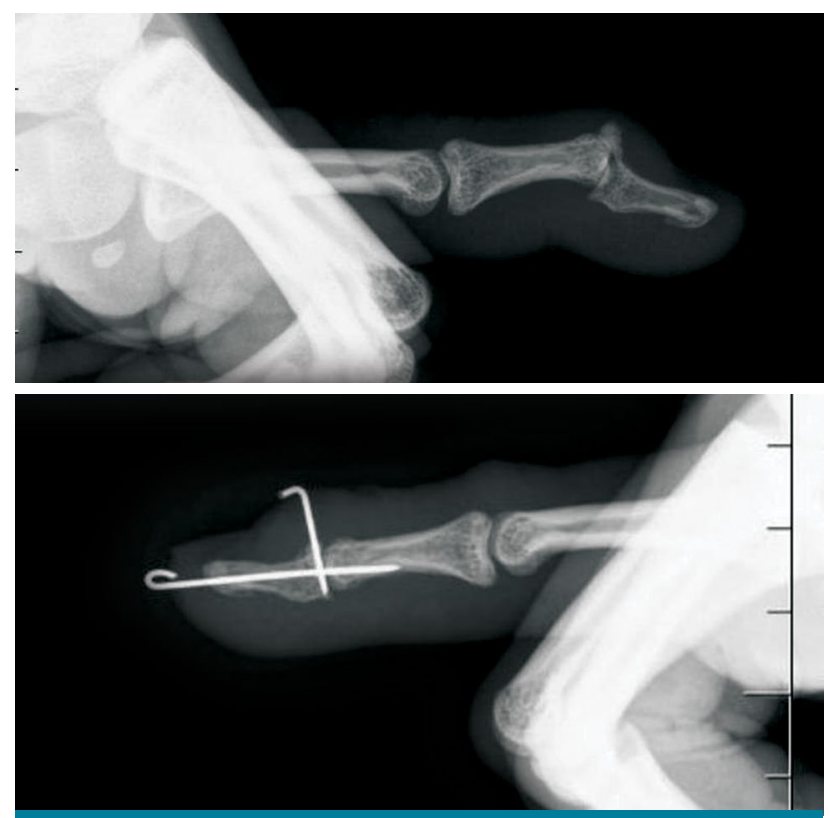

FIGURE 4. Direct fragment fixation method was preferred, when the fragment size was close to $50 \%$ of the joint surface.

\section{Statistical analysis}

Statistical analysis was performed using the SPSS version 25.0 software (IBM Corp., Armonk, NY, USA). Normality was tested using the Shapiro-Wilk test. As the data were not normally distributed, median (min-max) or interquartile range (IQR) values and number and frequency were used, where appropriate. The Wilcoxon signed-rank test was used to assess whether there was a significant difference in total range of motion (ROM) between the operated finger and the corresponding finger on the healthy side. A $p$ value of $<0.05$ was considered statistically significant.
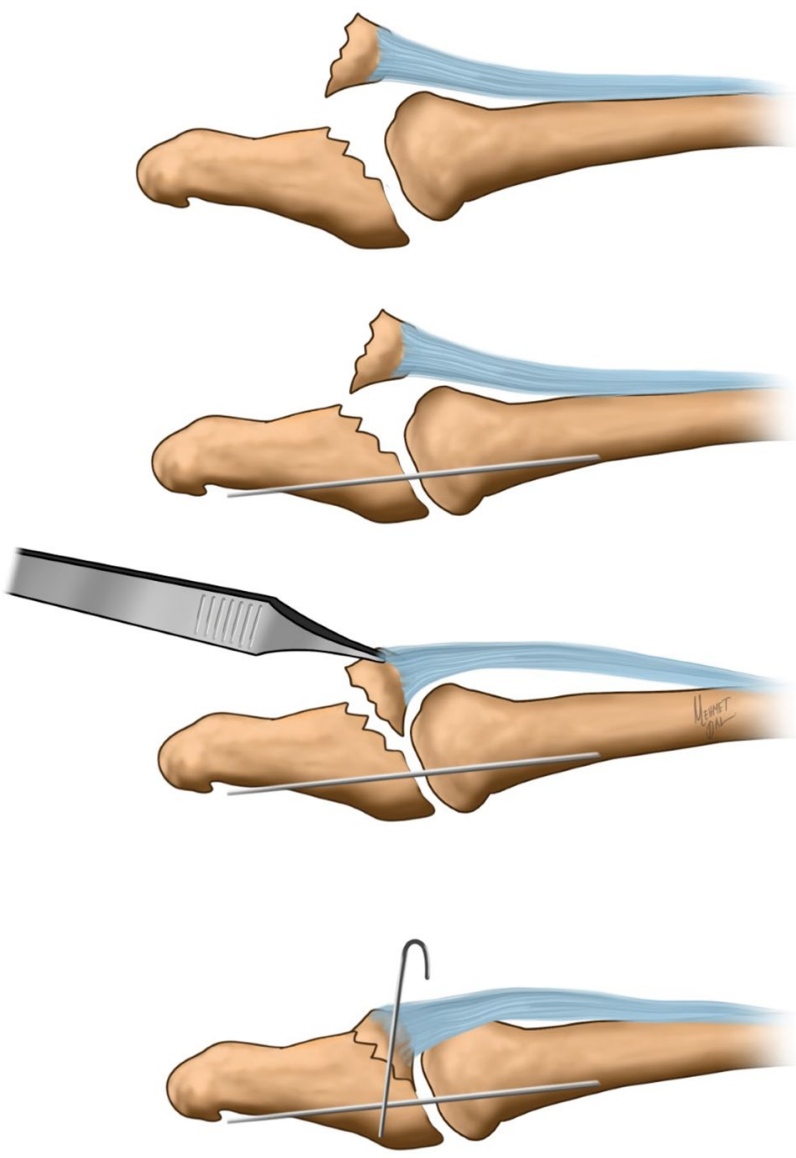

FIGURE 5. After the reduction of the DIP joint, a K-wire across the DIP joint was inserted volarly as much as possible, checking that it was not passing through the fracture line. The fragment was reduced with forceps and, then, fixed using a second $\mathrm{K}$-wire inserted from the dorsal to the volar side.

DIP: Distal interphalangeal; K-wire: Kirschner wire. 


\begin{tabular}{|c|c|c|c|c|}
\hline \multicolumn{5}{|c|}{$\begin{array}{l}\text { TABLE I } \\
\text { Demographic and clinical characteristics of patients }(n=19)\end{array}$} \\
\hline Baseline characteristics & $\mathrm{n}$ & $\%$ & Median & Min-Max \\
\hline Age (year) & & & 24.8 & $14-47$ \\
\hline Time to surgery (day) & & & 41 & $28-90$ \\
\hline Time to follow-up (month) & & & 9 & $3-50$ \\
\hline \multicolumn{5}{|l|}{ Sex } \\
\hline Male & 15 & 80 & & \\
\hline \multicolumn{5}{|l|}{ Affected finger } \\
\hline D2 & 2 & 10 & & \\
\hline D3 & 4 & 21 & & \\
\hline D4 & 5 & 26 & & \\
\hline D5 & 8 & 42 & & \\
\hline Dominant hand affected & 11 & 55 & & \\
\hline \multicolumn{5}{|c|}{ Wehbe and Schneider classification } \\
\hline Type I & 4 & 21 & & \\
\hline Type II & 15 & 79 & & \\
\hline \multicolumn{5}{|l|}{ Type of procedure } \\
\hline Dorsal block pin & 8 & 42 & & \\
\hline Umbrella handle & 3 & 16 & & \\
\hline Direct pinning & 8 & 42 & & \\
\hline
\end{tabular}

\section{RESULTS}

The median time from injury to surgery was 41 (range, 28 to 90) days. In 11 patients $(55 \%)$, the dominant hand was affected. The fifth finger was affected in eight patients, the fourth finger in five patients, the third finger in four patients, and the second finger in two patients. According to the Wehbé-Schneider classification, ${ }^{[20]}$ there were five type I and 14 type II mallet fingers.

According to Crawford criteria, outcomes were excellent in 11, good in four, fair in three, and poor in one patient. The median follow-up was 9.4 months (range, 3-50 months). The median DIP joint extension limitation was 7.63 (range, 0 to 40) degrees and the median ROM of the DIP joint was 66.3 (range, 20 to 90) degrees. There was no statistically significant difference in the postoperative ROM, compared to the uninjured side ( $p>0.05)$. Radiographic bone healing was achieved in all patients. No severe complications, such as skin necrosis, infection, malunion, nonunion, or K-wire breakage, were observed in any patients. Although two patients had mild degenerative changes on radiographs postoperatively, these changes did not limit their daily living activities. Only one patient had persistent mild pain during flexion and extension of the DIP joint.

\section{DISCUSSION}

The management of delayed bony mallet injury is complicated and still remains controversial. Therefore, the present study attempted to evaluate different methods applied according to the size of fracture fragments. Our results showed that successful clinical results were achieved in patients with delayed presentation of bony mallet finger via open reduction and internal fixation. ${ }^{[21]}$ The median DIP extension loss was 7.63 degrees, and the median ROM of the DIP joint was 66.3 degrees, and $79 \%$ of patients had good-to-excellent results. These findings are consistent with those of previous studies, showing high patient satisfaction rates. Tang et al. ${ }^{[13]}$ reported that the loss of extension was 0 to 10 degrees in 17 delayed cases with open reduction and compression with double K-wires technique. We believe that the removal of callus and fibrous tissue is necessary to anatomically reduce the fracture gap and palmar subluxation of the distal phalanx. Similarly, many surgeons recommend an open reduction to ensure contact between the bone surfaces. ${ }^{[11,12]}$ Reddy and $\mathrm{Ho}^{[22]}$ reported that open reduction not only provided anatomical reduction, but also released the adhesions of the extensor tendon to the surrounding tissue. 
In delayed cases, if the avulsed fragment is large, subluxation of the DIP joint may occur due to the continuous traction of the flexor tendon. ${ }^{[13]}$ However, there is no consensus in the literature regarding the critical size of a fragment that may lead to subluxation. In a biomechanical study, Husain et al. ${ }^{[23]}$ showed that, for subluxation to occur, the fragment size should be at least $43 \%$ of the joint surface. Giddins ${ }^{[24]}$ reported that instability was not solely a function of fracture fragment size and that there was a correlation between the pivoting of the fragment on extension testing and subsequent subluxation. Kim \& $\mathrm{Kim}^{[25]}$ showed that DIP subluxation was more likely, when the fracture fragment size was more than $48 \%$ of the base. Tang et al. ${ }^{[13]}$ reported that subluxation with a long injury time might lead to palmar contracture caused by the long-term flexion state of the DIP joint, and this situation complicated closed reduction. Chin and $\mathrm{Foo}^{[26]}$ recommended surgery, if the articular fragment was larger than $50 \%$, if the fragment could not be approximated with passive extension on fluoroscopy, or if DIP joint subluxation with hinging motion of volar fragment on the middle phalanx condyle was demonstrated on fluoroscopy.

Although multiple techniques have been proposed for delayed bone mallet finger, there is no consensus regarding the most optimal treatment modality. The main goals of treating bony mallet finger are to establish a congruent joint and to minimize extension lag. Since its first introduction by Ishiguro et al. ${ }^{[18]}$ in 1997, DBP has become one of the most popular techniques for the treatment of bony mallet finger. Although DBP is reliable and straightforward, it has some inherent disadvantages from DIP transfixation wire, such as articular cartilage damage and iatrogenic nail bed injury. ${ }^{[27]}$ Another issue with DBP is that it has poor control of the avulsed fracture block, and loss of fracture reduction may occur easily after surgery. Moreover, it is difficult to insert the transfixation pin volar to the fracture site, when the articular fragment is larger than $50 \%$. If the K-wire runs through the fracture site, anatomical reduction may not occur and fracture healing would be jeopardized. Due to these problems, Capkin et al. ${ }^{[28]}$ argued that the transfixation pin was not necessary and successful results could still be obtained only with DBP. In this study, they reported satisfactory clinical and radiological outcomes without any major complications. In our practice, we prefer two dorsal pins to prevent rotation of the fragment and to provide more stable fixation. In a recent study,
Polat et al. ${ }^{[29]}$ compared single and double dorsal wires in DBP, and found similar functional and clinical results between the two techniques.

Rocchi et al. ${ }^{[17]}$ proposed an umbrella handle method to reduce the fracture by pulling an umbrella handle-shaped K-wire from the palmar aspect of the finger. The results at eight weeks were evaluated as excellent in 11, good in 35, and fair in two patients. This technique does not require joint transfixation with piercing of the articular cartilage, and it allows immediate DIP joint motion. We consider that this method is more feasible, when the fragment is small and comminuted.

Badia and Riano ${ }^{[19]}$ described an interfragmentary fixation method in which a first K-wire was driven from the tip of the distal phalanx across the DIP joint to hold it in extension and a second wire was driven through the base of the distal phalanx and pulp. At an average follow-up period of 22 months, 16 patients had an average extension lag of 2 degrees and flexion of 75 degrees. Yamanaka and Sasaki ${ }^{[30]}$ used one or two compression pins to fix the dorsal fragment to the distal phalanx in 15 patients and obtained good results with 1 degree of hyperextension to 60 degrees of flexion. Also, Han et al. ${ }^{[31]}$ compared DBP and direct pinning and found that direct pinning was superior to DBP in terms of improvement in extensor lag and ROM; however, both groups achieved good results according to the Crawford criteria. In our practice, we prefer the direct pinning technique of Badia and Riano, ${ }^{[19]}$ when the fragment size is close to $50 \%$ of the joint surface, since it provides straightforward reduction of the DIP joint. Taken together, the most appropriate method should be selected depending on the size of the fracture fragment.

Several methods to evaluate the treatment results have been described in the literature. Most studies reporting mallet fingers use the Crawford classification, which is based on active extension deficit and pain. ${ }^{[15]}$ Accordingly, if the extension deficit is between 0 and 10 degrees, the result is considered excellent; if between 10 and 25 degrees, good; if more than 25 degrees, fair; and if the extension is painful, poor. In our study, we used the Crawford criteria, as it considers extension deficit, amount of flexion, and pain.

In a review of 24 studies, the DIP extension deficit was between 0 and 6.5 degrees. ${ }^{[32]}$ Although eight $(42 \%)$ of our patients had varying degrees of limitation of extension, only one of these patients had 
a poor result due to pain. Although final radiological results do not always correlate with clinical results, Warren et al. ${ }^{[33]}$ and others ${ }^{[32,34]}$ found no significant relationship between residual DIP joint extension deficit and patient satisfaction.

In the literature, the complication rate of surgical treatment ranges from 3 to $54 \% .{ }^{[35-37]}$ These complications are usually defined as nonunion, malunion, osteoarthritis, nail dystrophy, infection, and skin necrosis. Moreover, some authors recommend closed reduction, as skin and soft tissue complications may easily occur after open reduction. ${ }^{[35,36]}$ In a retrospective study, Stern and Kastrup $^{[37]}$ reviewed 123 mallet injuries and reported a complication rate of $53 \%$ in surgically treated patients. These complications included infections $(20 \%)$, permanent nail deformities $(18 \%)$, joint incongruity $(18 \%)$, fixation failure $(13 \%)$, and bony prominence $(11 \%)$. Our complication rate $(10 \%)$ was lower than the rates reported in the literature, and no complications were related to skin and soft tissues. Osteoarthritis was recognized in two patients, but was not considered painful. We believe that, with a meticulous open procedure, a low rate of soft tissue complications can be achieved.

The main limitations of our study are its singlecenter, retrospective design, small sample size, and the lack of control group. Since there are reports of successful non-operative treatment of bony mallet fractures in the literature, ${ }^{[20,38]}$ comparison with a conservative treatment group would decrease bias and add to the interpretability. Further large-scale, prospective, randomized studies are needed to elucidate the effectiveness of the delayed operative treatment options available for bony mallet finger.

In conclusion, with the open reduction technique, union can be achieved by providing anatomical reduction. In addition, extension lag can be eliminated in most patients by making the joint surface anatomical. The successful results with all three treatment methods indicate that there is no precise algorithm for the treatment of delayed bony mallet finger, and the most optimal method can be selected depending on the size of the fracture fragment.

\section{Declaration of conflicting interests}

The authors declared no conflicts of interest with respect to the authorship and/or publication of this article.

\section{Funding}

The authors received no financial support for the research and/or authorship of this article.

\section{REFERENCES}

1. Lamaris GA, Matthew MK. The diagnosis and management of mallet finger injuries. Hand (N Y) 2017;12:223-8.

2. Gumussuyu G, Asoglu MM, Guler O, May H, Turan A, Kose O. Extension pin block technique versus extension orthosis for acute bony mallet finger; a retrospective comparison. Orthop Traumatol Surg Res 2021;107:102764.

3. Damron TA, Engber WD. Surgical treatment of mallet finger fractures by tension band technique. Clin Orthop Relat Res 1994;(300):133-40.

4. Kronlage SC, Faust D. Open reduction and screw fixation of mallet fractures. J Hand Surg Br 2004;29:135-8.

5. Zhang X, Meng H, Shao X, Wen S, Zhu H, Mi X. Pull-out wire fixation for acute mallet finger fractures with k-wire stabilization of the distal interphalangeal joint. J Hand Surg Am 2010;35:1864-9.

6. Teoh LC, Lee JY. Mallet fractures: A novel approach to internal fixation using a hook plate. J Hand Surg Eur Vol 2007;32:24-30.

7. Miura T. Extension block pinning using a small external fixator for mallet finger fractures. J Hand Surg Am 2013;38:2348-52.

8. Moss JG, Steingold RF. The long term results of mallet finger injury. A retrospective study of one hundred cases. Hand 1983;15:151-4.

9. Chung DW, Lee JH. Anatomic reduction of mallet fractures using extension block and additional intrafocal pinning techniques. Clin Orthop Surg 2012;4:72-6.

10. Kootstra TJM, Keizer J, van Heijl M, Ferree S, Houwert M, van der Velde D. Delayed extension block pinning in 27 patients with mallet fracture. Hand (N Y) 2021;16:61-6.

11. Ulusoy MG, Karalezli N, Koçer U, Uysal A, Karaaslan O, Kankaya Y, et al. Pull-in suture technique for the treatment of mallet finger. Plast Reconstr Surg 2006;118:696-702.

12. Lee SK, Kim HJ, Lee KW, Kim KJ, Choy WS. Modified pullout wire suture technique for the treatment of chronic bony mallet finger. Ann Plast Surg 2010;65:466-70.

13. Tang J, Wu K, Wang J, Zhang J. Open reduction and compression with double Kirschner wires for the treatment of old bony mallet finger. J Orthop Surg Res 2019;14:459.

14. Handoll $\mathrm{HH}$, Vaghela MV. Interventions for treating mallet finger injuries. Cochrane Database Syst Rev 2004;(3):CD004574.

15. Crawford GP. The molded polythene splint for mallet finger deformities. J Hand Surg Am 1984;9:231-7.

16. Tang JB. Re: Levels of experience of surgeons in clinical studies. J Hand Surg Eur Vol 2009;34:137-8.

17. Rocchi L, Genitiempo M, Fanfani F. Percutaneous fixation of mallet fractures by the "umbrella handle" technique. J Hand Surg Br 2006;31:407-12.

18. Ishiguro T, Itoh $\mathrm{Y}$, Yabe $\mathrm{Y}$, Hashizume N. Extension block with Kirschner wire for fracture dislocation of the distal interphalangeal joint. Tech Hand Up Extrem Surg 1997;1:95-102.

19. Badia A, Riano F. A simple fixation method for unstable bony mallet finger. J Hand Surg Am 2004;29:1051-5.

20. Wehbé MA, Schneider LH. Mallet fractures. J Bone Joint Surg [Am] 1984;66:658-69.

21. Atik OŞ. Is there something new and interesting in my article? Eklem Hastalik Cerrahisi 2019;30:69. 
22. Reddy M, Ho CA. Comparison of percutaneous reduction and pin fixation in acute and chronic pediatric mallet fractures. J Pediatr Orthop 2019;39:146-52.

23. Husain SN, Dietz JF, Kalainov DM, Lautenschlager EP. A biomechanical study of distal interphalangeal joint subluxation after mallet fracture injury. J Hand Surg Am 2008;33:26-30.

24. Giddins GE. Bony mallet finger injuries: Assessment of stability with extension stress testing. J Hand Surg Eur Vol 2016;41:696-700.

25. Kim JK, Kim DJ. The risk factors associated with subluxation of the distal interphalangeal joint in mallet fracture. J Hand Surg Eur Vol 2015;40:63-7.

26. Chin YC, Foo TL. Tips and tricks in mallet fracture fixation. J Hand Surg Asian Pac Vol 2016;21:432-5.

27. Pegoli L, Toh S, Arai K, Fukuda A, Nishikawa S, Vallejo IG. The Ishiguro extension block technique for the treatment of mallet finger fracture: Indications and clinical results. J Hand Surg Br 2003;28:15-7.

28. Çapkın S, Buyuk AF, Sürücü S, Bakan OM, Atlihan D. Extension-block pinning to treat bony mallet finger: Is a transfixation pin necessary? Ulus Travma Acil Cerrahi Derg 2019;25:281-6.

29. Polat O, Bombaci H, Kibar B, Toy S. Comparison of single and double dorsal wires in the extension block technique for mallet fractures: Retrospective observational study. Medicine (Baltimore) 2021;100:e25419.
30. Yamanaka K, Sasaki T. Treatment of mallet fractures using compression fixation pins. J Hand Surg $\mathrm{Br}$ 1999;24:358-60.

31. Han HH, Cho HJ, Kim SY, Oh DY. Extension block and direct pinning methods for mallet fracture: A comparative study. Arch Plast Surg 2018;45:351-6.

32. Salazar Botero S, Hidalgo Diaz JJ, Benaïda A, Collon S, Facca S, Liverneaux PA. Review of acute traumatic closed mallet finger injuries in adults. Arch Plast Surg 2016;43:134-44.

33. Warren RA, Norris SH, Ferguson DG. Mallet finger: A trial of two splints. J Hand Surg Br 1988;13:151-3.

34. Gruber JS, Bot AG, Ring D. A prospective randomized controlled trial comparing night splinting with no splinting after treatment of mallet finger. Hand (N Y) 2014;9:145-50.

35. Hofmeister EP, Mazurek MT, Shin AY, Bishop AT. Extension block pinning for large mallet fractures. J Hand Surg Am 2003;28:453-9.

36. King HJ, Shin SJ, Kang ES. Complications of operative treatment for mallet fractures of the distal phalanx. J Hand Surg Br 2001;26:28-31.

37. Stern PJ, Kastrup JJ. Complications and prognosis of treatment of mallet finger. J Hand Surg Am 1988;13:329-34.

38. Thillemann JK, Thillemann TM, Kristensen PK, FoldagerJensen AD, Munk B. Splinting versus extension-block pinning of bony mallet finger: A randomized clinical trial. J Hand Surg Eur Vol 2020;45:574-81. 